\title{
Facile Deposition of Ultrafine Silver Particles on Silicon Surface Not Submerged in Precursor Solutions for Applications in Antireflective Layer
}

\author{
Bing Jiang, ${ }^{1}$ Meicheng Li, ${ }^{1,2}$ Dandan Song, ${ }^{1}$ Yingfeng Li, ${ }^{1}$ and Trevor Mwenya ${ }^{1}$ \\ ${ }^{1}$ State Key Laboratory of Alternate Electrical Power System with Renewable Energy Sources, School of Renewable Energy, \\ North China Electric Power University, Beijing 102206, China \\ ${ }^{2}$ Suzhou Institute, North China Electric Power University, Suzhou 215123, China \\ Correspondence should be addressed to Meicheng Li; mcli@ncepu.edu.cn
}

Received 22 January 2014; Revised 20 March 2014; Accepted 21 March 2014; Published 15 April 2014

Academic Editor: Yanbao Zhao

Copyright @ 2014 Bing Jiang et al. This is an open access article distributed under the Creative Commons Attribution License, which permits unrestricted use, distribution, and reproduction in any medium, provided the original work is properly cited.

\begin{abstract}
Using a facile deposition method, the ultrafine silver particles are successfully deposited on the Si surface that is not submerged in precursor solutions. The ultrafine silver particles have many advantages, such as quasiround shape, uniformity in size, monodisperse distribution, and reduction of agglomeration. The internal physical procedure in the deposition is also investigated. The results show that there are more particles on the rough Si surface due to the wetting effect of solid-liquid interface. The higher concentration of ethanol solvent can induce the increase of quantity and size of particles on Si surface not in solutions. The ultrafine particles can be used to prepare porous Si antireflective layer in solar cell applications.
\end{abstract}

\section{Introduction}

Metallic ultrafine particles $(1-100 \mathrm{~nm}$ in diameter) have attracted much attention for their anomalous physical properties which are different from the bulk solid phases [1]. The novel properties of ultrafine silver particles, such as surface-enhanced Raman spectroscopy (SERS), surface Plasmon renounce (SPR), and catalyst, have been exclusively studied [2-6]. Recently, many chemical methods have been used to synthesize silver particles [6-12]. But most of these methods focus on the synthesis of silver nanoparticles (AgNPs) instead of the one-step deposition methods on the certain substrate. Meanwhile, in most methods, the substrates need to be dipped into the precursor solutions. In vertical deposition process, the substrates are kept vertical to the surface of the solution, which can be used to prepare colloidal crystal and metal thin film or particles [13-17]. This method can produce the high-quality single crystal with controlled thickness by controlling the balance between ethanol evaporation rate and particle sedimentation [16]. The silver mirror reaction is an "old" chemical route, which can be used to generate reflective mirrors on solid supports. In recent times, studies of nanotechnology have renewed the interest for this reaction. The silver mirror reaction has been used to prepare silver particles and films, core-shell structures for extensive applications $[6,12,18-22]$. In this work, ultrafine silver particles are prepared on Si surface out of Tollen's reagent and the internal physical procedure of the deposition is revealed.

\section{Experimental Details}

Firstly, $\mathrm{AgNO}_{3}$ solutions were prepared in the mixed solvent of ethanol and water. The volume percent of ethanol was controlled at $0 \%, 50 \%$, and $70 \%$. The Tollen's reagent $(0.2 \mathrm{M})$ was obtained by dripping aqueous ammonia continuously into the $\mathrm{AgNO}_{3}$ solutions while stirring until the mixed solutions became transparent. Then, the rough and polished Si samples of $20 \times 10 \times 0.5 \mathrm{~mm}^{3}$ were vertically dipped into the Tollen's reagent, respectively. The depth of immerging was about $2 \mathrm{~mm}$, and the other part of the samples was out of solution. After that, the glucose $(0.01 \mathrm{M})$ was added to Tollen's reagent. In this typical silver mirror reaction, the $\mathrm{Ag}$ ions were reduced to $\mathrm{Ag}$ particles deposited on the $\mathrm{Si}$ 


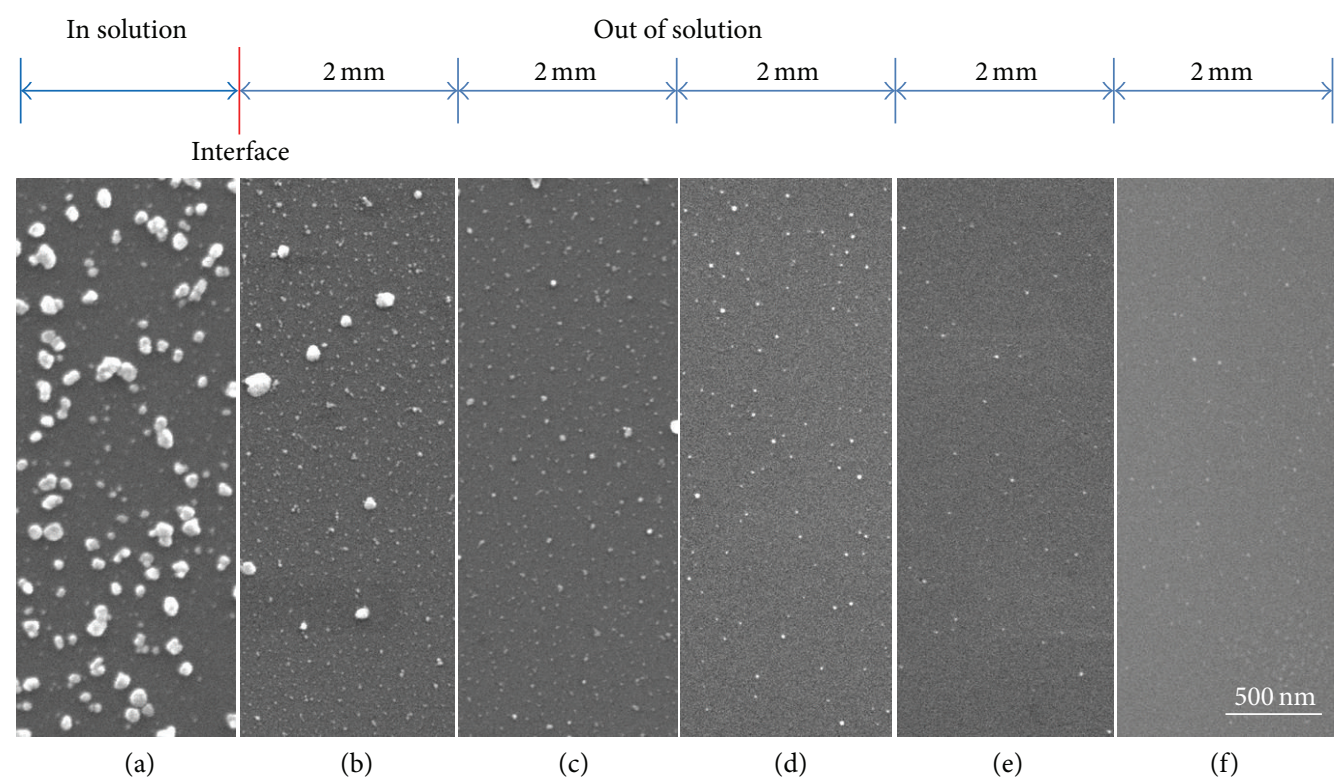

FIGURE 1: The changes of morphology of silver particles on surface in solutions and away from the solid-liquid interface after 5 minutes reaction (SEM images).

substrate. The reactions were kept for 2, 5, and 10 minutes at room temperature. The effect of reaction time, concentration of ethanol, and surface state (rough and polished) on $\mathrm{Ag}$ particles on the surface in solution and not in solution were investigated by scanning electron microscope (SEM, FEI Quanta 200F). Moreover, the polished Si wafer was put on horizontally above the reaction solutions (including 50\% ethanol solvent) away from liquid surface about $1 \mathrm{~mm}$. After that, the Ag nanoparticles on the Si surface above solutions were also investigated. Finally, the Si wafer deposited silver particles were immersed in etching solutions composed of $\mathrm{HF}, \mathrm{H}_{2} \mathrm{O}_{2}$, and $\mathrm{H}_{2} \mathrm{O}$ (volume ratio is $1: 5: 2$ ) for 5 minutes. Then, nanoporous $\mathrm{Si}$ antireflective layers were obtained by etching process. The morphology and antireflection property of porous Si were measured by SEM and ZoLix IPCE/QE system, respectively.

\section{Results and Discussion}

Through SEM observation, it is found that the ultrafine silver particles can be deposited on the surface out of solutions. Figure 1 shows the changes of morphology of the silver particles away from the solid-liquid interface after 5 minutes reaction. Compared with that on surface out of solution, there are more silver particles with the larger size on the surface in solution, as shown in Figures 1(a) and 1(b). It can be seen from Figures $1(\mathrm{c})-1(\mathrm{f})$ that the size and quantity of the ultrafine particles out of solutions display an obvious difference, which degrades with increase of distance away from the solidliquid interface. The size of particles in solution is about $50 \mathrm{~nm}$, whereas that out of solution is ranged from $5 \mathrm{~nm}$ to $20 \mathrm{~nm}$. The effective deposition distance out of solutions is about $1 \mathrm{~cm}$. It is considered that the successful deposition of ultrafine silver particles on the surface out of solutions is attributed to the wetting of solid-liquid interface. The wetting effect is related to the surface status and deposition process.

The wetting effect is proved by investigating the effect of the roughness on distribution of silver particles. Figure 2 shows the morphology of silver particles deposited on the polished and rough surfaces out of solutions by vertical deposition for $2 \mathrm{~min}$ and $5 \mathrm{~min}$. The result shows that, for 5 minutes reaction, the particles in polished surface are very small with size of 5-20 nm, while more particles with the larger size $(40-60 \mathrm{~nm})$ are deposited on the rough surfaces out of solutions. As is well known, the wetting of solid-liquid interface is closely correlated with surface roughness. The size and distribution of silver particles can be influenced by wetting force which depends on the surface state [15]. In our experiments, the nanoparticles deposited on rough surface are much more than those on the polished surface, which can be explained by the wetting effect during the vertical deposition. When a part of sample is immersed into the precursor solutions, the wetting effect makes a few precursor solutions being adsorbed slowly to the surface not submerged in solution, resulting in the successful preparation of ultrafine particles. These ultrafine particles may come from the particles of initial reaction in solutions or the subsequent reaction of precursor solutions on the surface out of solution.

In previous studies, the evaporation of ethanol solvent has been usually used to vertically deposit colloidal crystals [14]. In this work, the effect of ethanol solvent on deposition out of solutions is also investigated. Figure 3 shows that the quantity and size of particles on the surface out solution change by increasing the volume ratio of ethanol solvent. It is considered that the ultrafine Ag particles are taken 


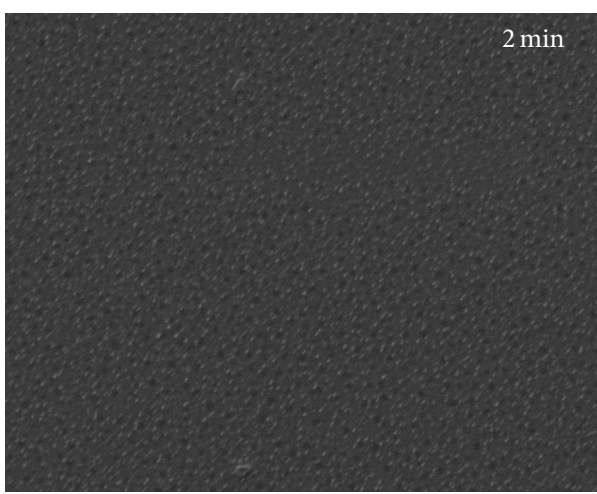

(a) Polished surface

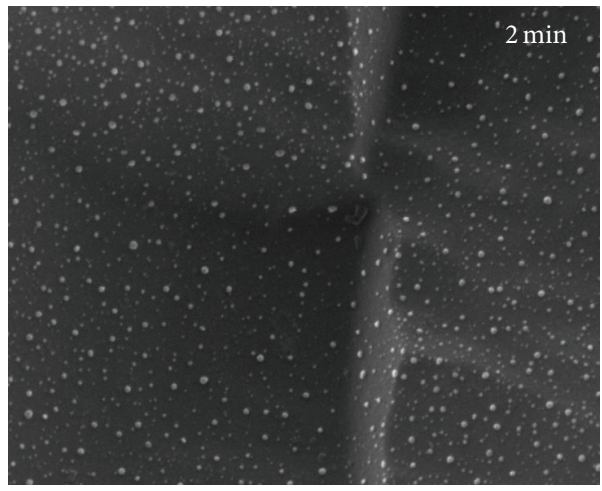

(c) Rough surface

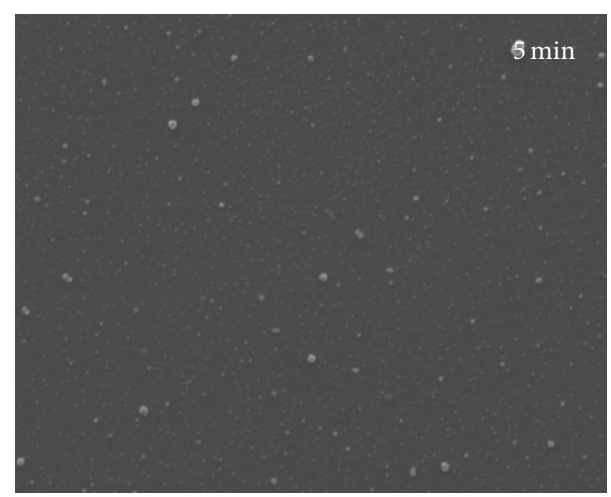

(b)

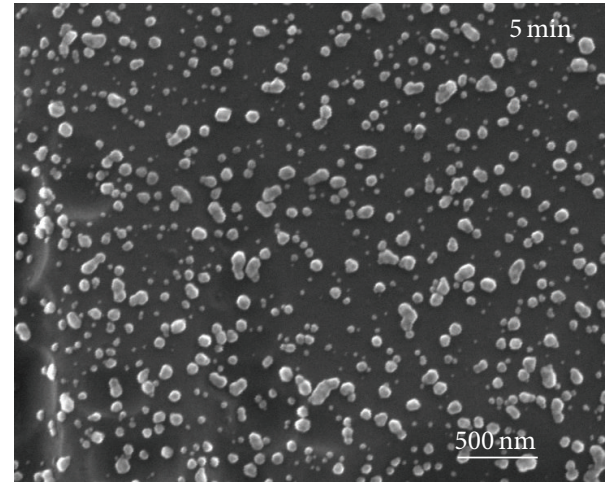

(d)

FIGURE 2: Morphology of silver particles deposited on the polished and rough surfaces out of solution by vertical deposition for (a) and (c) 2 minutes and (b) and (d) 5 minutes (SEM images).

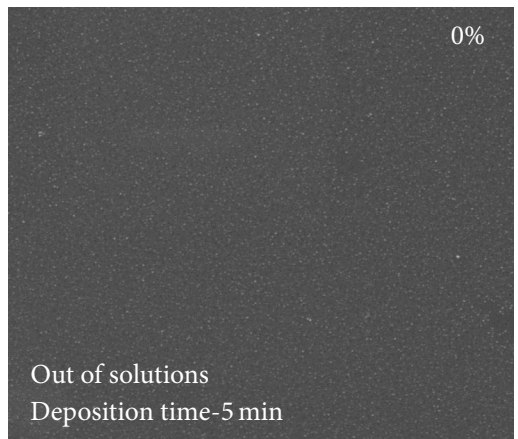

(a) Ethanol solvent

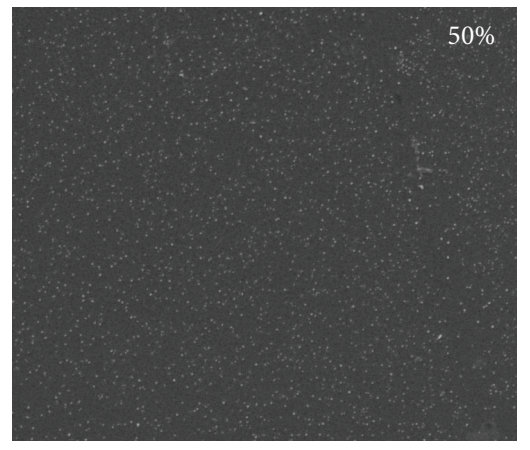

(b)

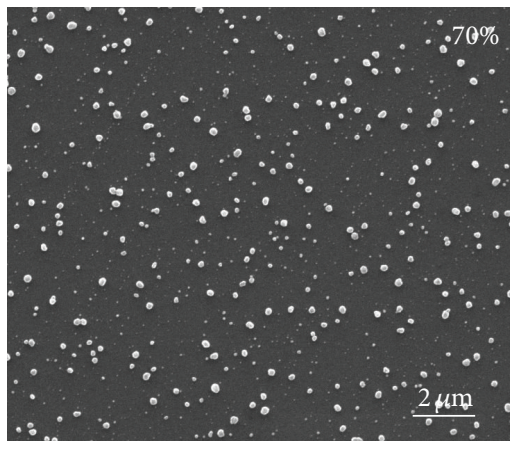

(c)

FIGURE 3: The changes of ultrafine particles deposited on the surface not in solutions under different concentration of ethanol. The volume ratio of ethanol solvent is (a) $0 \%$, (b) $50 \%$, and (c) $70 \%$ (SEM images).

out by the evaporation of ethanol solvent during vertical deposition. Moreover, it is found that, in the initial period of reaction, ultrafine Ag particles are more easily taken out of solution by evaporation. To prove the effect of ethanol solvent, we observe the experimental phenomenon when the sample is put horizontally above the solution away from liquid surface about $1 \mathrm{~mm}$. In this condition, a few ultrafine particles are observed on the sample surface not contacted with the solutions, but the distribution of particles is not uniform. The result proves that evaporation of ethanol solvent is another factor which contributed to the deposition of ultrafine particles on the surface out of solution. From the above results, it is found that the ultrafine particles are easily prepared on the surface not in solution and their size and shape are better controlled by the combined interaction of the wetting effect and evaporation of ethanol solvent.

Through investigation of deposition process, we find that the ultrafine silver particles can be obtained in the precursor solution with $50 \%$ ethanol solvent (volume ration). In the precursor solution with $70 \%$ ethanol solvent, the silver 


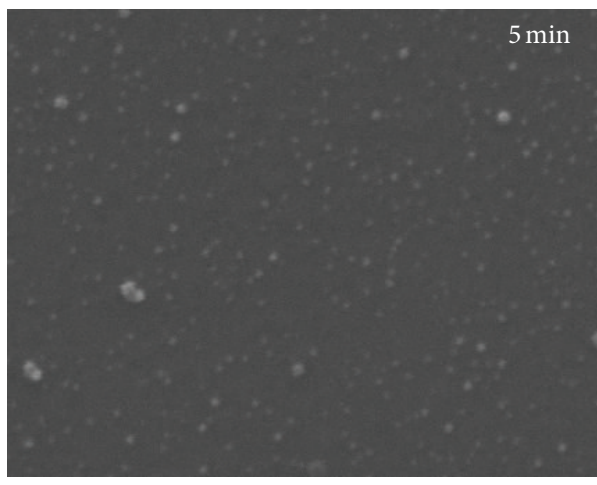

(a) Out of solutions

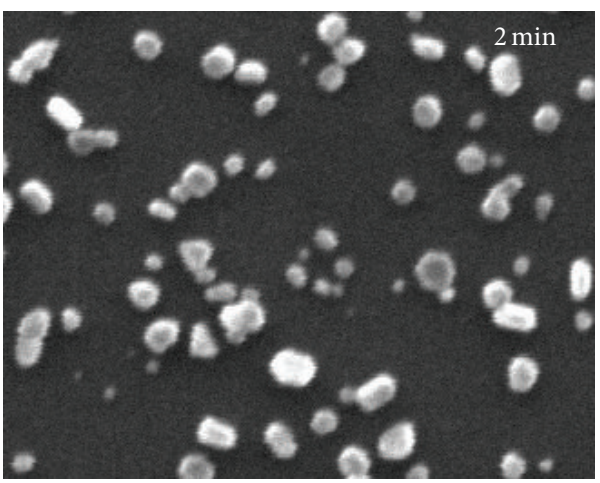

(c) In solutions

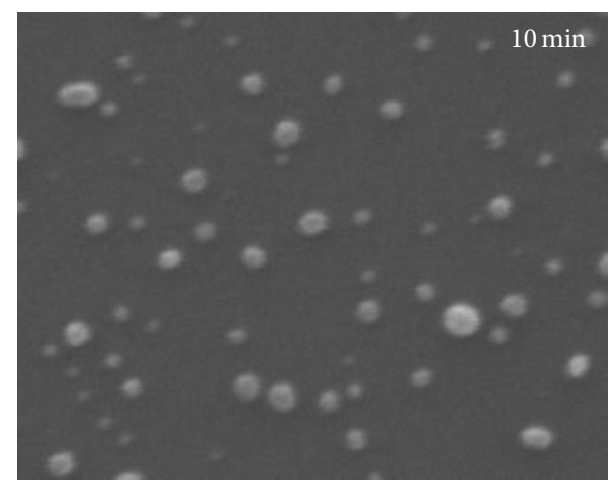

(b)

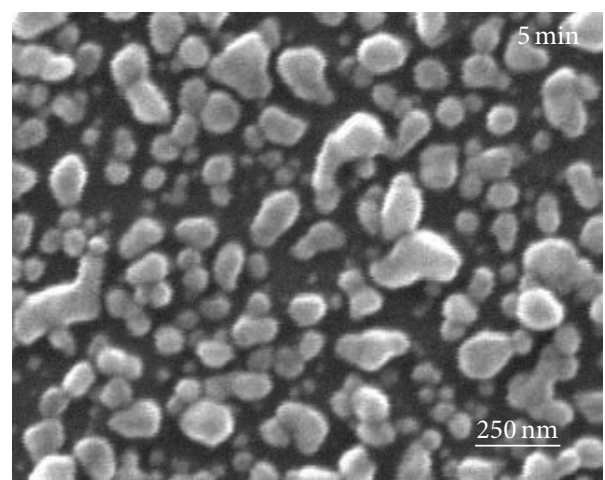

(d)

Figure 4: Morphology of silver particles on Si surface out of solutions and in solutions by vertical deposition. (a)-(b) out of solutions for 5 and 10 minutes; (c)-(d) in solutions for 2 and 5 minutes (SEM images).

particles are uniform but too large in size, whereas in solution without ethanol solvent, the silver particles are too little. Based on this, the effect of different reaction time on particles on the surface is also investigated, as shown in Figure 4. The result shows that the particles with the size of about 20-40 nm are obviously observed on the surface not in solutions (see Figures 4(a) and 4(b)), when the reaction time is $5 \mathrm{~min}$ and $10 \mathrm{~min}$. When the reaction time is short $(2 \mathrm{~min})$, the particles on the surface out of solutions are so little and small that they could be hardly observed. With the same reaction time, there are much more silver particles with larger sizes (50$100 \mathrm{~nm}$ ) on the surface in solutions, as shown in Figures $4(\mathrm{c})$ and $4(\mathrm{~d})$. The quantity and size of particles increase with reaction time, regardless of whether the surface is in solution or not. Compared with the quasiround ultrafine silver particles on surface out of solutions, the particles on the surface in solutions display irregular shape, nonuniform distribution, and agglomeration.

In the present work, the ultrafine silver particles are used to prepare porous $\mathrm{Si}$ antireflective layer. The result shows that ultrafine porous $\mathrm{Si}$ is obtained by catalytic etching ultrafine silver particles, which has smaller and more uniform ultrafine holes. The reflectance of ultrafine porous $\mathrm{Si}$ in near infrared waveband range is decreased to below $1.6 \%$ by the ultrafine porous Si structure, as shown in Figure 5. The improvement of antireflection is attributed to better light scattering of ultrafine porous Si due to the increase of the

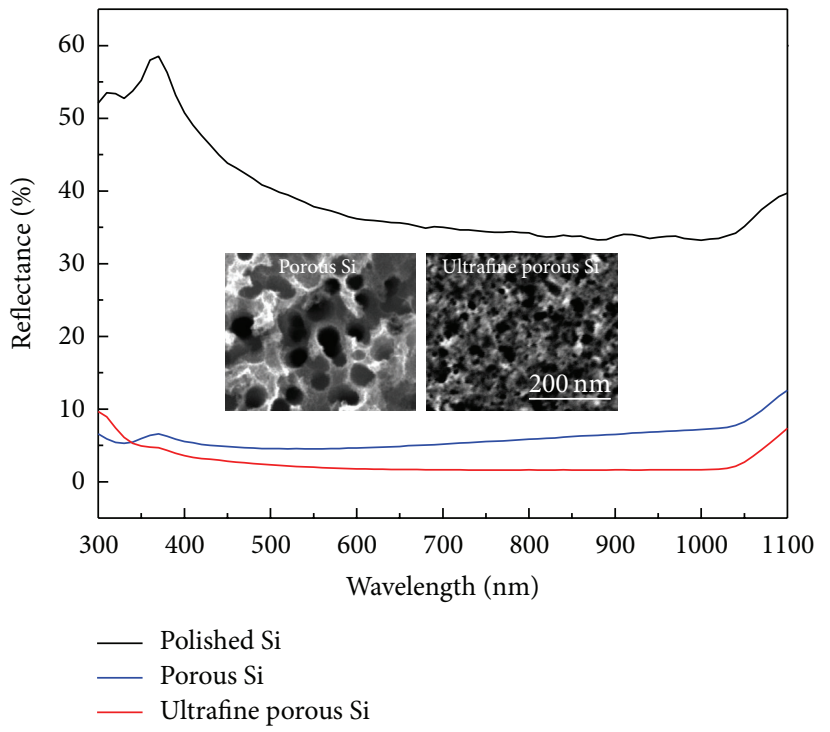

FIGURE 5: The antireflection property of porous Si and ultrafine porous $\mathrm{Si}$ etched by silver particles on the surface in solutions and not in solutions. The insets show the morphology of the porous $\mathrm{Si}$ and ultrafine porous Si.

optical absorption path lengths $[23,24]$. The ultrafine porous Si will be used to antireflective layer in solar cell devices. 


\section{Conclusion}

In this work, we propose a facile vertical deposition, which can be used to prepare ultrafine silver particles on the surfaces that can not be entirely dipped into the precursor solutions. The experimental results prove that the wetting effect of solid-liquid interface and evaporation of ethanol solvent are the internal physical mechanism in vertical deposition. The wetting effect is more obvious in the rough surface out of solution. Meanwhile, the many larger silver particles are obtained by increasing the concentration of ethanol solvent. Through process optimization, ultrafine silver particles with $10-40 \mathrm{~nm}$ in size can be deposited on the surface not in solution. The ultrafine porous Si antireflective layer can be obtained by etching ultrafine silver particles, which has the lower reflectance in a broader spectral range.

\section{Conflict of Interests}

The authors declare that there is no conflict of interests regarding the publication of this paper.

\section{Acknowledgments}

The authors acknowledge support of National Natural Science Foundation of China (Grant nos. 91333122, 51372082, 51202067, 51172069, and 50972032), Ph.D Programs Foundation of Ministry of Education of China (20130036110012 and 20110036110006), and Fundamental Research Funds for the Central Universities (11ZG02 and 12QN15).

\section{References}

[1] K. Ito, I. Tsuyumoto, A. Harata, and T. Sawada, "Ultrafast energy transfer of silver ultrafine particles in aqueous solutions as investigated by the ultrafast lensing effect technique," Chemical Physics Letters, vol. 318, no. 1-3, pp. 1-6, 2000.

[2] Z. Xu, J. Hao, F. Li, and X. Meng, "Surface-enhanced Raman spectroscopy of arsenate and arsenite using Ag nanofilm prepared by modified mirror reaction," Journal of Colloid and Interface Science, vol. 347, no. 1, pp. 90-95, 2010.

[3] H. Dai, M. Li, Y. Li, H. Yu, F. Bai, and X. Ren, "Effective light trapping enhancement by plasmonic Ag nanoparticles on silicon pyramid surface," Optics Express, vol. 20, pp. A502A509, 2012.

[4] Y. Saito, J. J. Wang, D. N. Batchelder, and D. A. Smith, "Simple chemical method for forming silver surfaces with controlled grain sizes for surface plasmon experiments," Langmuir, vol. 19, no. 17, pp. 6857-6861, 2003.

[5] X. Geng, M. Li, L. Zhao, and P. W. Bohn, "Metal-assisted chemical etching using Tollen's reagent to deposit silver nanoparticle catalysts for fabrication of quasi-ordered silicon micro/nanostructures," Journal of Electronic Materials, vol. 40, no. 12, pp. 2480-2485, 2011.

[6] B. Jiang, M. Li, F. Bai et al., "Morphology-controlled synthesis of silver nanoparticles on the silicon substrate by a facile silver mirror reaction," AIP Advances, vol. 3, no. 3, Article ID 032119, 2013.
[7] Y. Sun and Y. Xia, "Shape-controlled synthesis of gold and silver nanoparticles," Science, vol. 298, no. 5601, pp. 2176-2179, 2002.

[8] B. Wiley, Y. Sun, B. Mayers, and Y. Xia, "Shape-controlled synthesis of metal nanostructures: the case of silver," Chemistry A, vol. 11, no. 2, pp. 454-463, 2005.

[9] Y. Xia, Y. Xiong, B. Lim, and S. E. Skrabalak, "Shape-controlled synthesis of metal nanocrystals: simple chemistry meets complex physics?" Angewandte Chemie, vol. 48, no. 1, pp. 60-103, 2009.

[10] G. A. Martínez-Castañón, N. Niño-Martínez, J. P. LoyolaRodríguez, N. Patiño-Marín, J. R. Martínez-Mendoza, and F. Ruiz, "Synthesis of silver particles with different sizes and morphologies," Materials Letters, vol. 63, no. 15, pp. 1266-1268, 2009.

[11] Q. Zhang, W. Li, L.-P. Wen, J. Chen, and Y. Xia, "Facile synthesis of $\mathrm{Ag}$ nanocubes of 30 to $70 \mathrm{~nm}$ in edge length with $\mathrm{CF}$ 3COOAg as a precursor," Chemistry A, vol. 16, no. 33, pp. 1023410239, 2010.

[12] B. Jiang, M. Li, D. Song, Y. Li, and T. Mwenya, "A facile direct deposition of silver nanoparticles on silicon surface by silver mirror process," Crystal Research and Technology, vol. 48, no. 12, pp. 1044-1049, 2013.

[13] P. Jiang, J. F. Bertone, K. S. Hwang, and V. L. Colvin, "Singlecrystal colloidal multilayers of controlled thickness," Chemistry of Materials, vol. 11, no. 8, pp. 2132-2140, 1999.

[14] L. M. Goldenberg, J. Wagner, J. Stumpe, B.-R. Paulke, and E. Görnitz, "Ordered arrays of large latex particles organized by vertical deposition," Langmuir, vol. 18, no. 8, pp. 3319-3323, 2002.

[15] J. J. Diao, F. S. Qiu, G. D. Chen, and M. E. Reeves, "Surface vertical deposition for gold nanoparticle film," Journal of Physics D: Applied Physics, vol. 36, no. 3, pp. L25-L27, 2003.

[16] S.-L. Kuai, X.-F. Hu, A. Haché, and V.-V. Truong, "High-quality colloidal photonic crystals obtained by optimizing growth parameters in a vertical deposition technique," Journal of Crystal Growth, vol. 267, no. 1-2, pp. 317-324, 2004.

[17] B. Jiang, M. Li, Y. Li, D. Song, and T. Mwenya, "Vertical deposition of ultrafine silver particles on silicon surface out of solutions by silver mirror process," Materials Letters, vol. 116, pp. 195-198, 2014.

[18] D. Yu and V. W.-W. Yam, "Hydrothermal-induced assembly of colloidal silver spheres into various nanoparticles on the basis of HTAB-modified silver mirror reaction," Journal of Physical Chemistry B, vol. 109, no. 12, pp. 5497-5503, 2005.

[19] Z. Chen, X. Chen, L. Zheng et al., "A simple and controlled method of preparing uniform Ag midnanoparticles on Tollenssoaked silica spheres," Journal of Colloid and Interface Science, vol. 285, no. 1, pp. 146-151, 2005.

[20] R. Dondi, W. Su, G. A. Griffith, G. Clark, and G. A. Burley, "Highly size- and shape-controlled synthesis of silver nanoparticles via a templated tollens reaction," Small, vol. 8, no. 5, pp. 770-776, 2012.

[21] X. Li, J. Shen, A. Du et al., "Facile synthesis of silver nanoparticles with high concentration via a CTAB-induced silver mirror reaction," Colloids and Surfaces A: Physicochemical and Engineering Aspects, vol. 400, pp. 73-79, 2012.

[22] R. G. Shimmin, A. J. DiMauro, and P. V. Braun, "Slow vertical deposition of colloidal crystals: a langmuir-blodgett process?" Langmuir, vol. 22, no. 15, pp. 6507-6513, 2006. 
[23] C.-H. Chang, P. Yu, M.-H. Hsu et al., "Combined micro-and nano-scale surface textures for enhanced near-infrared light harvesting in silicon photovoltaics," Nanotechnology, vol. 22, no. 9, Article ID 095201, 2011.

[24] P. Ferrand and R. Romestain, "Optical losses in porous silicon waveguides in the near-infrared: effects of scattering," Applied Physics Letters, vol. 77, no. 22, pp. 3535-3537, 2000. 

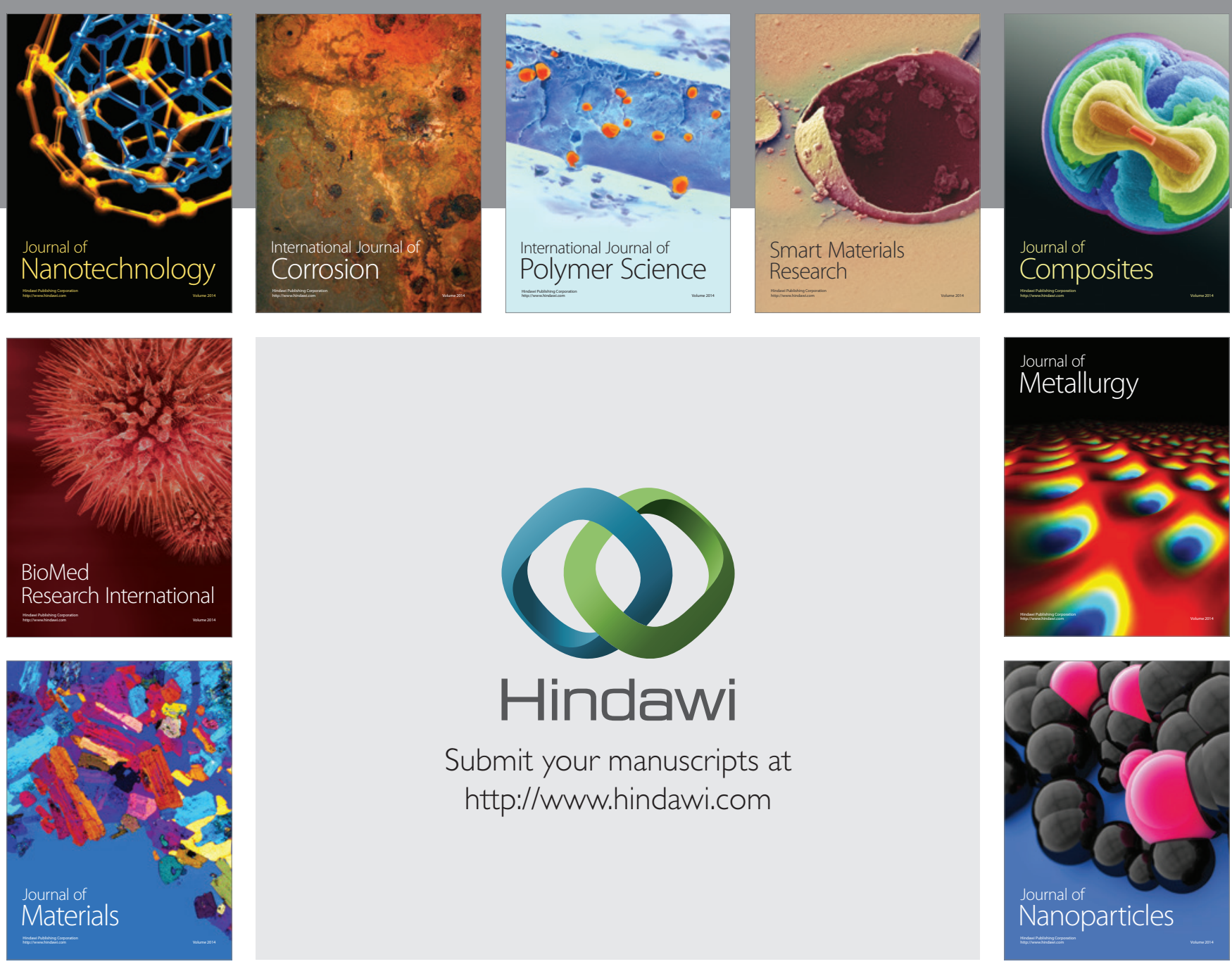

Submit your manuscripts at http://www.hindawi.com
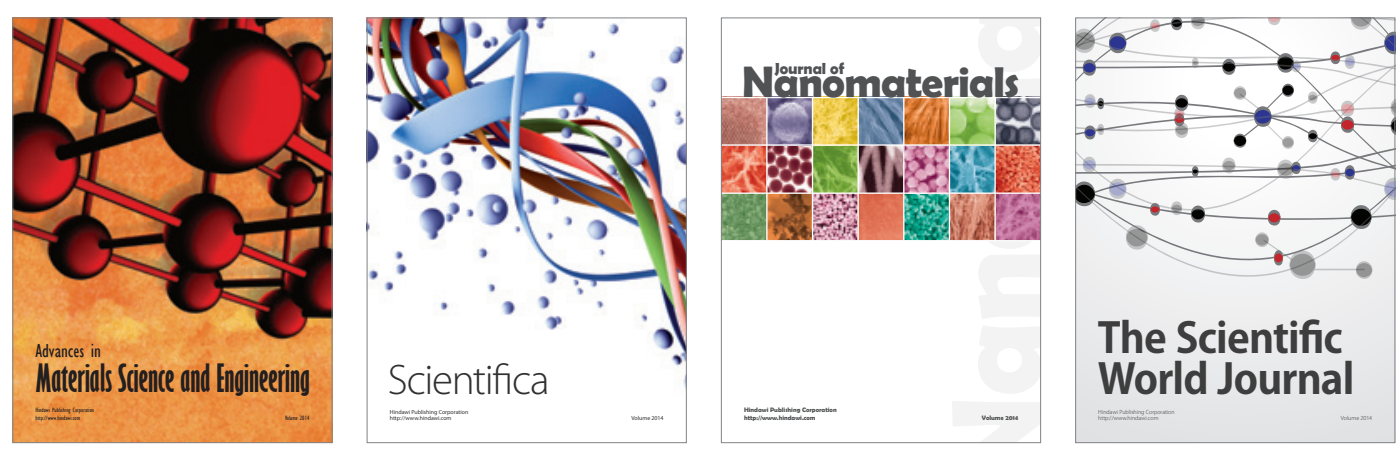

\section{The Scientific World Journal}
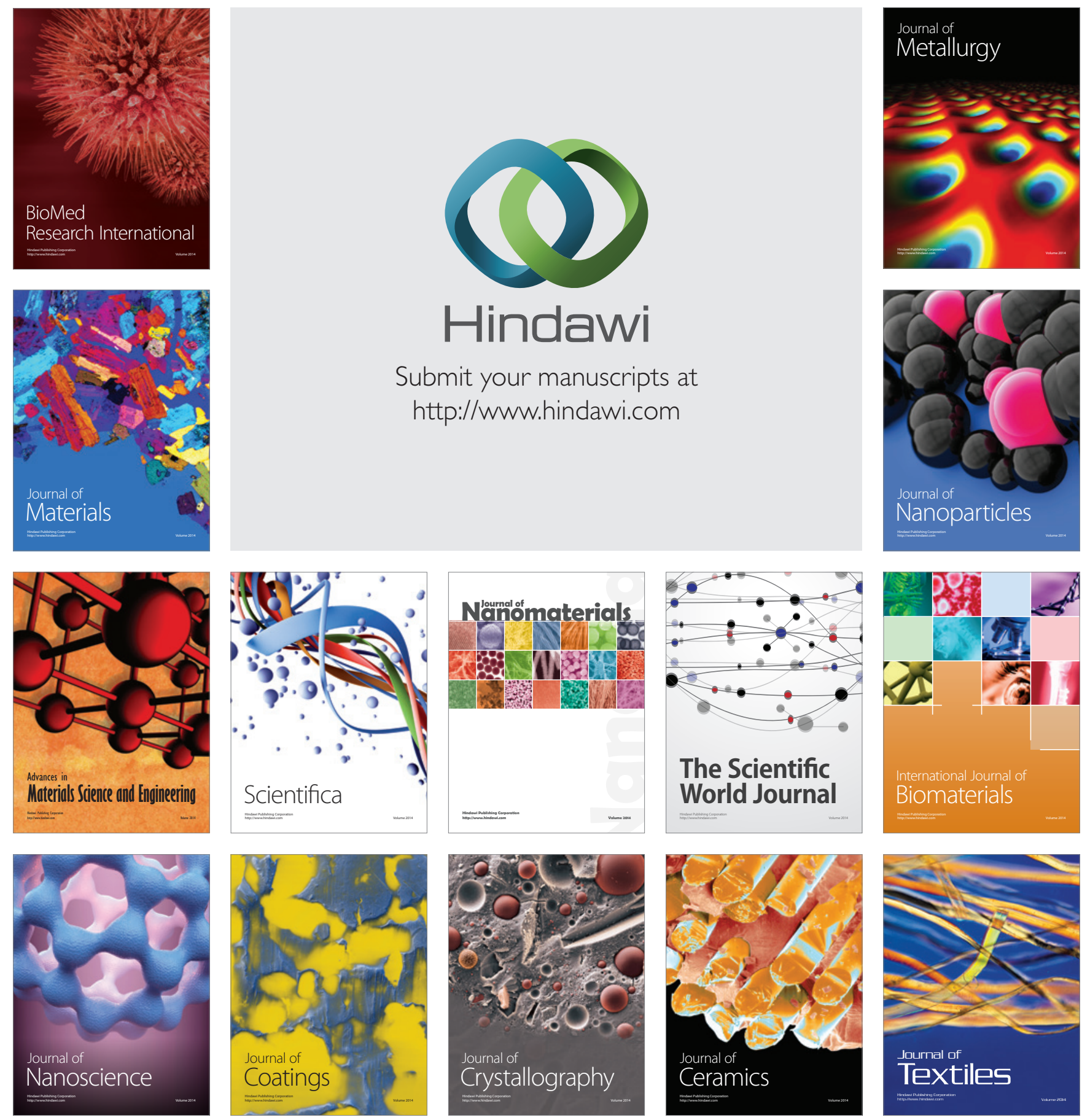